\title{
Non-muscle-invasive bladder cancer surveillance for which cystoscopy is partly BJUI replaced by microsatellite analysis of urine: a cost-effective alternative?
}

\author{
Esther W. de Bekker-Grob, Madelon N.M. van der Aa*, Ellen C. Zwarthoff', \\ Marinus J.C. Eijkemans, Bas W. van Rhijn*, Theo H. van der Kwast ${ }^{+\dagger}$ and \\ Ewout W. Steyerberg
}

Departments of Public Health, *Urology, and ${ }^{+}$Pathology, Erasmus MC, Rotterdam, Netherlands, and ${ }^{*}$ Department of Pathology and Laboratory, University Health Network and Mount Sinai Hospital, Toronto, Canada

Accepted for publication 23 October 2008

Study Type - Economic (Markov model with sensitivity analysis)

Level of Evidence $2 b$

\section{OBJECTIVE}

To determine how good microsatellite analysis (MA) markers in voided urine samples should be to make a surveillance procedure cost-effective in which cystoscopy is partly replaced by MA for patients with non-muscle-invasive urothelial carcinoma (NMI-UC).

\section{PATIENTS AND METHODS}

We constructed a semi-Markov model with a time horizon of 2 years, and a man aged 65 years as reference case. Data were used from a randomized trial (including 448 patients with NMI-UC from 10 hospitals), and from other data sources. The costs and effects (probability of being in a specific health state) were compared for two surveillance strategies: (i) cystoscopy of the urinary bladder every 3 months (conventional arm), and (ii) semi-automated MA of voided urine samples to identify loss of heterozygosity every 3 months, with a control cystoscopy at 3,12 and 24 months (test arm). Various sensitivity analyses were used to determine the sensitivity, specificity, and costs of MA of urine for which the test arm was as cost-effective as the conventional arm.

\section{RESULTS}

The probability of being without recurrence after 2 years of surveillance was similar ( $86.6 \%$ conventional arm vs $86.3 \%$ test arm) with currently available MA markers (sensitivity of $58 \%$ and specificity of $73 \%$ ). However, the test arm led to higher costs
(€4104 vs €3433 per head). The test arm would be as effective and cost the same as the conventional arm if the sensitivity of the currently available MA markers was increased at $\geq 61 \%$, had a specificity of $73 \%$, and decreased the costs of the MA test per follow-up sample from $€ 158$ to $<€ 70$.

\section{CONCLUSIONS}

Over 2 years, surveillance in which cystoscopy is partly replaced by currently available urinary MA to reduce patient burden can only provide a cost-effective alternative to the conventional surveillance if the MA urine test had a slightly higher sensitivity and its costs could be reduced.

\section{KEYWORDS}

cost-effectiveness, urine test, Markov decision model, microsatellite analysis, surveillance, urothelial carcinoma

\section{INTRODUCTION}

In the Western world bladder cancer is the fourth most common malignancy among men, after prostate, lung and colon cancers [1]. Histologically, 90-95\% of bladder cancers in the western world are urothelial carcinoma (UC). UC not only originate from the urothelial lining of the bladder, but they can also occur in the pyelum, the ureters or the urethra. Clinically, a distinction is made between nonmuscle-invasive (NMI) and muscle-invasive (MI) UC. NMI-UC are generally treated surgically by a transurethral resection (TUR). Although these NMI-UC are generally rather indolent, their recurrence rate is high (up to $75 \%$ ). Close monitoring of patients with a NMI-UC is warranted as $10-20 \%$ of recurrent tumours can show progression to MI UC; the latter is a life-threatening disease, with a 5year survival of $\approx 50 \%$, even after curative surgery (i.e. cystectomy). Surveillance of patients with NMI-UC is by regular cystoscopy (combined with cytological examination for high-risk NMI-UC). Although cystoscopy is considered as the reference standard for detecting tumour recurrences, a disadvantage of the method is its inability to detect recurrences in the upper urinary tract (UUT, pyelum, ureter). Moreover, cystoscopy is an invasive procedure, that can be associated with subsequent complaints of anxiety, dysuria and UTI, adversely influencing the quality of life of the patients [2-4]. Thus substantial efforts have been undertaken to design strategies that could reduce the frequency of cystoscopy in patients with NMI-UC $[5,6]$. One of these strategies is to replace cystoscopy partly by microsatellite 
FIG. 1. Interventions in the conventional and test arm of the randomized study, in which the plus sign indicates a positive MA test.

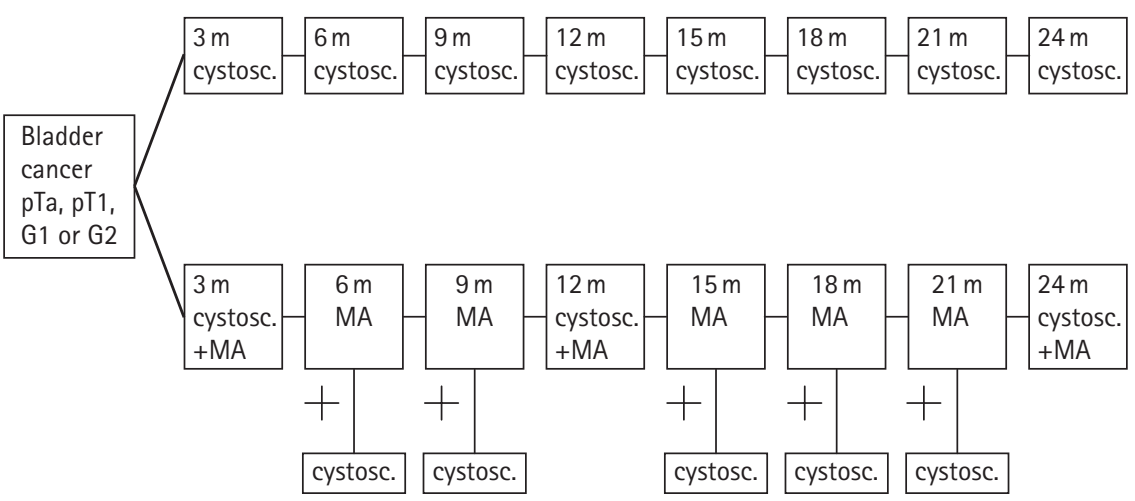

analysis (MA) to identify loss of

heterozygosity in urine samples; published

reports show that $M A$ is a highly sensitive and specific marker for diagnosing and monitoring UC, especially in patients with low-stage and low-grade tumours [7]. Van der Aa et al. [8] showed, in a randomized controlled trial, that MA can be used to detect tumours earlier and to detect tumours in the UUT that might be missed by cystoscopy. However, the sensitivity (58\%) and specificity of MA (73\%) for detecting recurrences in the bladder were both worse than for cystoscopy (98\% and 88\%, respectively) [8].

In the present study we determined the requirements in terms of sensitivity, specificity and real costs of MA markers on voided urine samples to achieve costeffectiveness of a surveillance procedure of patients with NMI-UC whose cystoscopy is partly replaced by MA. We used data from the randomised controlled Cost-effectiveness of Follow-up of urinary Bladder Cancer (CEFUB) trial.

\section{PATIENTS AND METHODS}

We conducted the randomized controlled CEFUB trial (including 448 patients with NMIUC, recruited by 10 Dutch hospitals; registration ClinicalTrials.gov NCT00126958) to evaluate the efficacy of MA on voided urine for detecting tumour recurrences in the follow-up of patients. Patients included had a primary or recurrent NMI-UC (pTa, pT1, G1 or G2) of the urinary bladder, based on histopathological examination of the surgically removed tumour. Figure 1 shows the interventions in the conventional and test arm of the randomized study over 2 years. The conventional arm consisted of cystoscopy of the urinary bladder every 3 months. The test arm consisted of semi-automated MA of DNA (for 20 polymorphic microsatellite markers on 10 chromosomes) extracted from voided urine samples from patients with a NMI-UC every 3 months. A blood sample was taken as a control for the MA of the subsequent urine samples, and was used during the entire study period. A control cystoscopy was done at 3, 12 and 24 months after TUR, and if the MA was positive. If a tumour recurrence was found by cystoscopy, irrespective of conventional or test arm, it was removed by TUR. If there was progression to MI-UC or G3 NMI-UC after adjuvant intravesical treatment, a cystectomy was considered. Additional radio-imaging was used to examine the UUT for the presence of $U C$, if the clinical performance was suspect or if the outcome of MA and cystoscopy were discordant, i.e. MA was positive but no tumour was detected by cystoscopy during two subsequent visits (i.e. a persistently positive MA). The UUT radio-imaging was not done immediately for a positive MA and negative cystoscopy, as previous studies showed that a positive MA test frequently antedates cystoscopically detectable recurrences $[8,9]$. Finally, 80 radio-imaging procedures showed seven tumours in the UUT (five in the test arm and two in the control arm). If there was suspected UC in the UUT, a unilateral nephroureterectomy was considered.

We constructed a semi-Markov decision model to assess costs and effects (probability of being in a specific health state) of both bladder cancer surveillance methods (i.e. the test and conventional arm). The model was
FIG. 2. The core of the model for bladder cancer surveillance: monitoring patients with NMI-UC can prevent progression to $\mathrm{MI}-\mathrm{UC}$ by detecting and treating recurrent tumours first (i.e. it is possible to prevent progression). This figure was used for the construction of the Markov model.

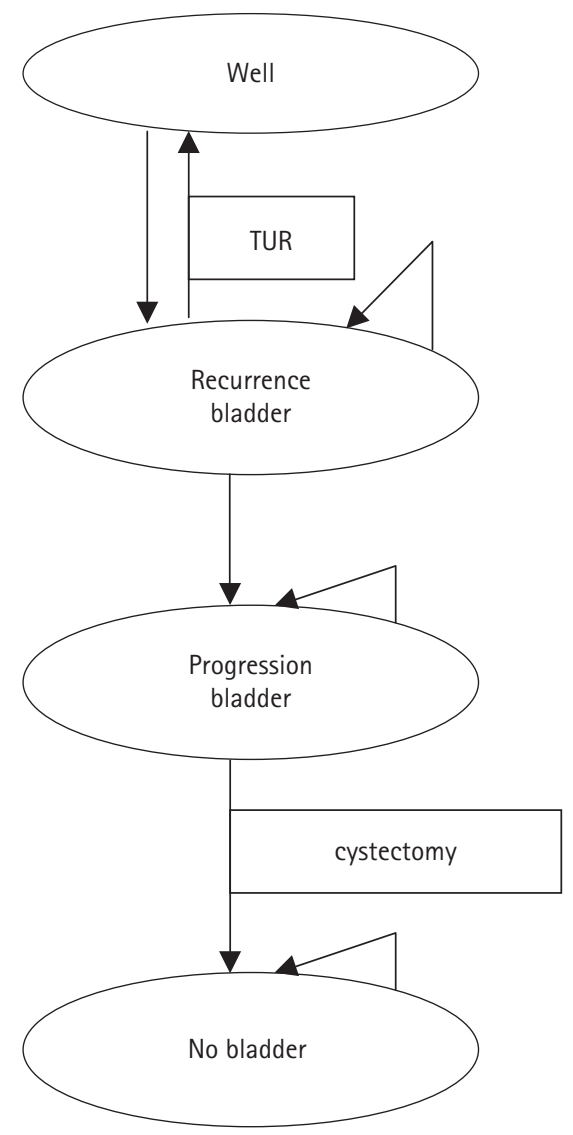

implemented in DATA software (Version TreeAge PRO 2007, TreeAge Software, Boston, MA, USA). Figure 2 highlights the core of the surveillance model; monitoring patients with $\mathrm{NMI-UC}$ can prevent progression to MI-UC by detecting and treating recurrent tumours first (i.e. it is possible to prevent progression).

Appendix 1 shows the underlying conceptual model; missing those patients with recurrent tumours can lead to cystectomy and a higher probability of death due to progressive tumours. Poor health states indicate dead, no bladder, progression (i.e. MI-UC or G3 NMI$\mathrm{UC}$ ) and recurrent $\mathrm{UC}$, and all other health states indicate 'well'. Notably, after a detected recurrent $U C$, the patient will go back to the health state 'well' again. Transition probabilities for each 3 months were used to correspond with the follow-up times. The reference case was a man aged 65 years, and the time horizon was 2 years. To retain some 
TABLE 1 The baseline assumptions of the Markov model

\begin{tabular}{|c|c|c|c|}
\hline Description & $\begin{array}{l}\text { Sensitivity/specificity, } \\
\% \text { or } P \text { or costs, } €\end{array}$ & Note & Source \\
\hline Sensitivity MA, bladder & 58 & & study \\
\hline Sensitivity MA, UUT & 58 & & study \\
\hline Specificity MA, bladder & 73 & & study \\
\hline Specificity MA, UUT & 73 & & study \\
\hline Sensitivity cystoscopy, bladder & 98 & & study \\
\hline Sensitivity cystoscopy, UUT & 0 & & study \\
\hline Specificity cystoscopy, bladder & 88 & & study \\
\hline Specificity cystoscopy, UUT & 100 & & study \\
\hline Sensitivity IVU & 60 & & http://www.guideline.gov \\
\hline $\begin{array}{l}\text { Specificity IVU } \\
\text { 3-month, } P\end{array}$ & 100 & False positives not possible & \\
\hline \multirow[t]{2}{*}{ Dead after progression } & 0.12 & 3-month risk year 1 & Dutch comprehensive cancer centres \\
\hline & 0.085 & 3-month risk year 2 & Dutch comprehensive cancer centres \\
\hline Progression after recurrence & 0.157 & & study \\
\hline Recurrence & 0.058 & $\begin{array}{l}\text { Selection primary tumour }+ \\
\text { counted for } 3 \text { recurrences }\end{array}$ & study \\
\hline Recurrence UUT & 0.1 & Given there is a recurrence & Dutch comprehensive cancer centres \\
\hline Recurrence bladder after NU & 0.12 & & [10] \\
\hline \multicolumn{4}{|l|}{ Costs } \\
\hline MA blood analysis & 357 & & Department of Pathology, Erasmus MC \\
\hline MA urine analysis & 158 & & Department of Pathology, Erasmus MC \\
\hline Cystoscopy & 168 & & Department of Finance, AMC \\
\hline IVU & 165 & & Department of Finance, AMC \\
\hline TUR & 1409 & & Department of Finance, AMC \\
\hline Cystectomy & 7997 & & Department of Finance, AMC \\
\hline Nephroureterectomy & 3333 & & Department of Finance, AMC \\
\hline
\end{tabular}

memory in the Markov model some health states were constructed with a 'sub-health' state, indicating whether the MA was positive and the cystoscopy negative at the last visit of surveillance. When this was also the case in the subsequent visit, an IVU was assumed to be done.

Table 1 shows the variables used in the Markov model to provide insight into the current effects and costs of both strategies [10]. Data from the trial (448 patients) were used to determine the 3-month probabilities of recurrence $\left(P_{\text {rec }}=.058\right)$ and progression after recurrence of NMI bladder cancer $\left(P_{\text {prog }}=0.157\right)$ [8]. Also, the 3-month probabilities of undergoing a cystoscopy, sensitivity (58\%) and specificity (73\%) of MA, and sensitivity and specificity of cystoscopy in the bladder ( $98 \%$ and $88 \%$, respectively) were determined by our trial data. Detailed, integral cost data of the MA analysis (blood and urine analysis) were obtained from the Department of Pathology of the Erasmus Medical Centre Rotterdam. Cost data for cystoscopy, IVU, TUR, cystectomy, and unilateral nephroureterectomy were obtained from the Department of Finance of the Academic Medical Centre Amsterdam. Unit prices were determined by following the microcosting method [11], which is based on comprehensive 'bottom-up' analyses and included costs of employment, material and equipment. A societal perspective was adopted. Costs of one cystoscopy procedure were calculated as $€ 168$ per patient (the including personal costs were relatively low (€63.4 per patient) due to the short diagnostic time and efficient organization in the participating hospitals). We compared these costs with the determined unit prices of the Department of Finance of Erasmus Medical Centre Rotterdam. The costs were about the same. Confidentiality agreements did not allow us to give more detailed information. Costs of a single MA blood analysis and MA urine analysis were, respectively, $€ 357$ and $€ 158$. MA of the blood acted as reference value for MA urine analysis. Therefore, during 2 years of surveillance, costs of MA blood analysis were estimated only once (i.e. all costs were at the beginning of the surveillance). However, the costs of MA urine analysis were incurred at every follow-up.

We made the following assumptions in the present study: (i) progression takes place after recurrence; (ii) only one recurrence can take place at the same time; (iii) the health state 'recurrence in UUT after nephroureterectomy' does not exist; this prevents a second possible nephroureterectomy in the model; (iv) only patients who had progression of the tumour at a specific time have a higher probability of death than the background mortality; (v) a cystoscopy never reveals a progressive recurrence, which originated in the UUT and invades the bladder; (vi) UUT imaging has a specificity of 100\%; (vii) in case of progression to MI-UC the next step was a cystectomy; (viii) after cystectomy the surveillance stops; and (ix) if the IVU was positive the next step was a nephroureterectomy.

One-way sensitivity analyses were used to evaluate the effects of varying costs, recurrence rate, progression rate, sensitivity rates, and specificity rates. All point estimates 


\begin{tabular}{|c|c|c|c|c|c|c|c|c|c|}
\hline \multirow[b]{2}{*}{$\begin{array}{l}\text { Stage, } \\
\text { months }\end{array}$} & \multicolumn{2}{|c|}{ Costs, $€$} & \multicolumn{7}{|l|}{$\%$} \\
\hline & /stage & Cumulative & Dead & $\begin{array}{l}\text { No } \\
\text { bladder }\end{array}$ & $\begin{array}{l}\text { Progression } \\
\text { UUT }\end{array}$ & $\begin{array}{l}\text { Progression } \\
\text { bladder }\end{array}$ & $\begin{array}{l}\text { Recurrence } \\
\text { UUT }\end{array}$ & $\begin{array}{l}\text { Recurrence } \\
\text { bladder }\end{array}$ & Well \\
\hline \multicolumn{10}{|c|}{ Conventional } \\
\hline 3 & 451 & 451 & 0.37 & 0.80 & 0.09 & 0.02 & 0.49 & 0.09 & 98.15 \\
\hline 6 & 447 & 897 & 0.75 & 1.61 & 0.25 & 0.02 & 0.89 & 0.09 & 96.40 \\
\hline 9 & 440 & 1337 & 1.15 & 2.40 & 0.44 & 0.02 & 1.21 & 0.09 & 94.69 \\
\hline 12 & 433 & 1769 & 1.57 & 3.18 & 0.66 & 0.02 & 1.48 & 0.08 & 93.01 \\
\hline 15 & 426 & 2195 & 1.99 & 3.94 & 0.92 & 0.02 & 1.70 & 0.08 & 91.35 \\
\hline 18 & 419 & 2615 & 2.43 & 4.68 & 1.19 & 0.02 & 1.87 & 0.08 & 89.73 \\
\hline 21 & 412 & 3027 & 2.89 & 5.41 & 1.47 & 0.02 & 2.01 & 0.08 & 88.13 \\
\hline 24 & 406 & 3433 & 3.37 & 6.12 & 1.74 & 0.01 & 2.12 & 0.08 & 86.57 \\
\hline \multicolumn{10}{|l|}{ Test } \\
\hline 3 & 964 & 964 & 0.37 & 0.80 & 0.09 & 0.02 & 0.49 & 0.09 & 98.15 \\
\hline 6 & 336 & 1300 & 0.75 & 1.27 & 0.21 & 0.36 & 0.76 & 1.91 & 94.75 \\
\hline 9 & 355 & 1655 & 1.18 & 2.05 & 0.34 & 0.60 & 0.98 & 3.37 & 91.47 \\
\hline 12 & 713 & 2367 & 1.66 & 3.81 & 0.46 & 0.04 & 1.11 & 0.11 & 92.81 \\
\hline 15 & 323 & 2690 & 2.06 & 4.26 & 0.59 & 0.35 & 1.21 & 1.85 & 89.68 \\
\hline 18 & 346 & 3036 & 2.50 & 5.02 & 0.70 & 0.58 & 1.27 & 3.22 & 86.72 \\
\hline 21 & 366 & 3402 & 2.97 & 6.00 & 0.79 & 0.76 & 1.29 & 3.93 & 84.27 \\
\hline 24 & 702 & 4104 & 3.45 & 7.94 & 0.86 & 0.04 & 1.30 & 0.11 & 86.29 \\
\hline
\end{tabular}

of these variables were halved or doubled, except the point estimates of the sensitivity and specificity rates, which were increased and decreased based on previous reports $[8,12]$.

Finally, by using a two-way sensitivity analysis between the sensitivity and specificity of MA, we determined a threshold line at which both surveillance strategies were equally effective. Various combinations of MA sensitivity, specificity, and costs of MA urine analysis were determined in which the test arm was as effective and cost the same as the conventional arm.

\section{RESULTS}

Table 2 shows the current costs and effects based on the Markov model for the conventional and test arm, respectively, for different follow-up samples. Over 2 years the total costs of surveillance were lower ( $€ 3433$ per head, €1283 diagnostic and €2150 therapeutic costs) for the conventional arm, than for the test arm (€4104 per head, €2347 diagnostic and $€ 1757$ therapeutic costs; column 3, stage 24 months). For the conventional arm the cost per head decreased by every subsequent stage (column 2), because the probability to be in health states 'Dead' (column 4) or 'No bladder' (column 5) increased over the time. For the test arm the costs per head at stage 3 months were more than twice as high as the conventional arm. At stage 3 months not only was there MA urine analysis, but also a MA blood analysis and cystoscopy. Stages 12 and 24 months were more expensive than the conventional arm due to the control cystoscopy.

Over the 2 years there was a probability of $6.1 \%$ of losing the bladder in the conventional arm and $7.9 \%$ in the test arm (see column 5, stage 24 months). The probability of having a progressive tumour in the bladder was very low, but higher $(0.04 \%)$ in the test arm than the conventional arm $(0.01 \%$; column 7 , stage 24 months). However, the test arm resulted in better outcomes for UC originating in the UUT due to using IVU after twice-discordant outcomes between MA and cystoscopy in subsequent visits. Although the test arm detected tumours earlier and in the UUT, more tumours in the bladder were missed.

Therefore, the current conventional and the test arm were similarly effective over the 2 years (Fig. 3 ). Thus, the Markov model suggested, as expected, that the conventional arm dominated the test arm in terms of costeffectiveness. The conventional arm had lower costs and a slightly more patients in a good health state (86.6\% vs $86.3 \%$; Table 2 , column 10, stage 24 months). Figure 3 also showed that (i) if patients had only MA with
FIG. 3. The probability (\%) of being in a poor health state in either surveillance strategy (conventional or test arm) for different times of follow-up. Poor health states indicate dead, no bladder, progression and recurrence of $U C$.

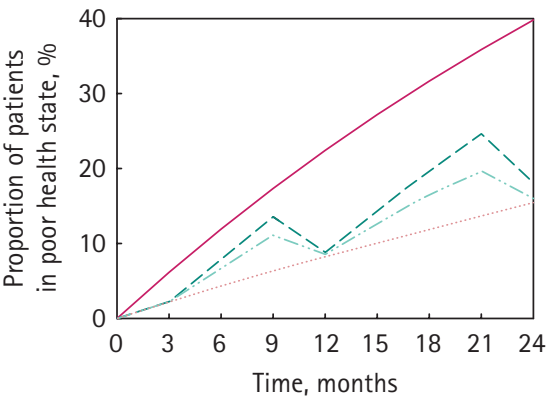

\begin{tabular}{|l}
\hline No surveillance \\
Conventional surveillance (every \\
3 months cystoscopy) \\
---- Less invasive conventional surveillance \\
(cystoscopy at 3,12 , and 24 months) \\
$-\cdots-$ Test arm (every 3 months MA with \\
control cystoscopy at 3, 12, and 24 \\
months)
\end{tabular}

no control cystoscopy at stage 3,12 and 24 months, the probability of being in a poor health state would increase steeply; and (ii) if patients had only a cystoscopy at stage 3,12 and 24 months with no MA, fewer would be in a good health state than in the test arm. 


\begin{tabular}{|c|c|c|c|c|c|c|c|}
\hline \multirow[b]{2}{*}{ Variable } & \multirow[b]{2}{*}{ Variability } & \multicolumn{2}{|c|}{ Cumulative costs, $€$} & \multirow{2}{*}{$\begin{array}{l}\text { Difference } \\
\text { test - con }\end{array}$} & \multicolumn{2}{|c|}{ Probability, $\%$ of being 'well' } & \multirow{2}{*}{$\begin{array}{l}\text { Difference } \\
\text { test - con }\end{array}$} \\
\hline & & $\overline{\text { Test }}$ & Conventional & & $\overline{\text { Test }}$ & Conventional & \\
\hline \multirow[t]{2}{*}{ Recurrence rate } & 0.029 & 3629 & 3053 & $576^{*}$ & 91.48 & 91.69 & -0.21 \\
\hline & 0.116 & 4954 & 4135 & $819^{*}$ & 77.04 & 77.08 & -0.04 \\
\hline \multirow[t]{2}{*}{ Progression rate } & 0.079 & 3905 & 3276 & $629^{*}$ & 90.17 & 89.52 & $0.65 t$ \\
\hline & 0.314 & 4440 & 3731 & $709^{*}$ & 79.65 & 80.94 & -1.29 \\
\hline \multirow[t]{2}{*}{ Costs cystoscopy, $€$} & 84 & 3745 & 2792 & $953^{*}$ & 86.29 & 89.57 & -3.28 \\
\hline & 336 & 4823 & 4716 & $107^{*}$ & 86.29 & 86.57 & -0.28 \\
\hline \multirow[t]{2}{*}{ Costs MA blood, $€$} & 179 & 3926 & 3433 & $493^{*}$ & 86.29 & 89.57 & -3.28 \\
\hline & 714 & 4460 & 3433 & $1027^{*}$ & 86.29 & 86.57 & -0.28 \\
\hline \multirow[t]{2}{*}{ Costs MA urine, $€$} & 79 & 3502 & 3433 & $69^{*}$ & 86.29 & 89.57 & -3.28 \\
\hline & 316 & 5308 & 3433 & $1875^{*}$ & 86.29 & 86.57 & -0.28 \\
\hline \multirow[t]{2}{*}{ Sensitivity MA } & 0.29 & 4079 & 3433 & $646^{*}$ & 83.87 & 86.57 & -2.70 \\
\hline & 0.70 & 4111 & 3433 & $678^{*}$ & 87.27 & 86.57 & $0.70 t$ \\
\hline \multirow[t]{2}{*}{ Specificity MA } & 0.69 & 4181 & 3433 & $748^{*}$ & 86.30 & 86.57 & -0.27 \\
\hline & 0.76 & 4048 & 3433 & $615^{*}$ & 86.28 & 86.57 & -0.29 \\
\hline \multirow[t]{2}{*}{ Sensitivity cystoscopy } & 0.84 & 4093 & 3432 & $661^{*}$ & 84.63 & 85.31 & -0.68 \\
\hline & 0.98 & 4104 & 3433 & $671^{*}$ & 86.29 & 86.57 & -0.28 \\
\hline \multirow[t]{2}{*}{ Specificity cystoscopy } & 0.79 & 4583 & 4327 & $256^{*}$ & 86.29 & 86.57 & -0.28 \\
\hline & 0.98 & 3572 & 2439 & $1133^{*}$ & 86.29 & 86.57 & -0.28 \\
\hline
\end{tabular}

*Test arm is more expensive than conventional arm over 2 years; +Test arm is more effective than conventional arm over 2 years.

FIG. 4. The threshold analysis for surveillance strategies. Sensitivity and specificity combinations in the white area indicate that the conventional arm is more effective; sensitivity and specificity combinations in the light green area means that the test arm is more effective.

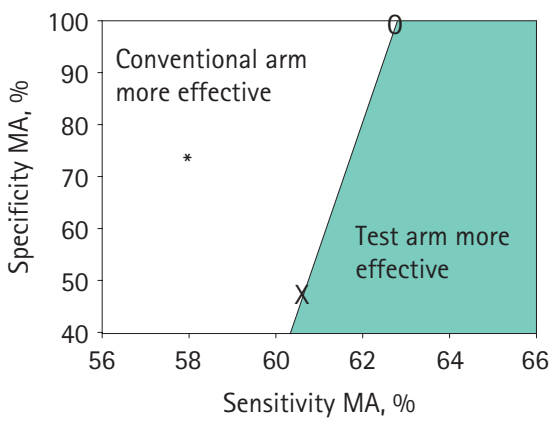

* = current assumption for MA test (MA sensitivity $58 \%$, MA specificity $73 \%$ )

$x=$ if MA-urine test has a sensitivity of $60.5 \%$, a specificity of $47 \%$, and will be free of charge, the test arm will be as effective and cost the same as the conventional arm

$0=$ if MA-urine test has a sensitivity of $62.8 \%$ and a specificity of $100 \%$, and will cost $€ 130.5$, the test arm will be as effective and cost the same as the conventional arm

Table 3 shows various one-way sensitivity analyses of costs and effects for both surveillance arms over the 2 years. Changing the variables over plausible ranges did not

TABLE 4 Combinations of MA sensitivity, specificity, and costs of MA urine in which the test arm is as
effective and costs the same as the conventional arm
\begin{tabular}{lcc} 
Sensitivity MA, \% & Specificity MA, \% & Costs of MA urine, $€$ \\
\hline 60.5 & 47 & 0.0 \\
60.7 & 53 & 17.0 \\
61.4 & 73 & 69.6 \\
62.8 & 100 & 130.5
\end{tabular}

make the test arm cheaper than the conventional arm. Only if the progression rate was halved (i.e. 0.079) was the probability of being 'Well' (i.e. being without a recurrence or progressive UC) larger in the test arm than the conventional arm (90.2\% vs $89.5 \%$, respectively) over the 2 years. This was also the case if the sensitivity of MA was increased to $70 \%$ (i.e. probability of being 'Well' was $87.3 \%$ in the test arm vs $86.6 \%$ in the conventional arm). Obviously, changing the costs did not influence the health effect side.

Figure 4 shows that the test arm was equally effective as the conventional arm, if the MA had a sensitivity of $60.3 \%$ in combination with a specificity of $39.8 \%$, or if the MA had a sensitivity of $62.8 \%$ in combination with a specificity of $100 \%$, or all linear combinations of MA sensitivity and specificity in between. Table 4 shows that, for instance, if the sensitivity was increased to $61 \%$, the specificity of MA remained at $73 \%$, and the costs of MA urine tests will decrease to $€ 70$, then the test arm is as effective and costs the same as the conventional arm.

\section{DISCUSSION}

Currently available MA markers are not sensitive enough (sensitivity of 58\%) or specific (specificity of 73\%) to use as a replacement for cystoscopy in the surveillance of bladder cancer. The probability of being without recurrence after 2 years of surveillance was similar for both surveillance strategies (86.6\% conventional arm vs $86.3 \%$ test arm), but the test arm led to higher costs (€4104 vs €3433 per head). The conventional arm hence dominated the test arm (less costs, similar effects). However, the test arm will be 
as effective and cost the same as the conventional arm if the sensitivity of the current available MA markers increased to $\geq 61 \%$, the specificity of MA remained at $73 \%$ and the costs of MA urine test per follow-up sample decreased from $€ 158$ to $<€ 70$.

Lotan and Roehrborn [13] used a decision tree to evaluate the cost-effectiveness of substituting cystoscopy by other bladder tumour markers than MA markers. They showed that using a urine-based tumour marker alternating with cystoscopy is costeffective for a wide range of marker sensitivities, specificities and costs, a 20-80\% yearly recurrence and a 2-40\% yearly progression rate. Although the sensitivity and specificity of their tumour markers and our MA markers were almost the same as in the present study (respectively, 50\% vs 58\%, and $70 \%$ vs $73 \%$ ), the costs of diagnostic and therapeutic interventions used in both studies differed, especially for the costs of the tumour markers. Ignoring the inflation rate, the costs of the tumour markers were assumed as $\$ 50$ [13], in contrast to $€ 158$ for our MA urine analysis and $€ 357$ for the one-time MA blood analysis. It is plausible that genetic analysis (i.e. MA markers) are more expensive than urine-sediment diagnosis. However, for a fair comparison of costs we should use the same costing method. The costs in the present study were based on unit prices determined by following the microcosting method [11], while the costs in Lotan and Roehrborn [13] were according to the listing of the manufacturers. Another important difference between both studies is that in the present study at stage 3,12 and 24 months there was a control cystoscopy in addition to MA urine analysis, which increased the costs.

Our Markov model did not explicitly account for the burden (i.e. quality of life, e.g. pain, infection rate) associated with cystoscopy. Cystoscopy would take place 7.6 times in the conventional arm and just 4.3 times in the test arm over the 2 years. However, we assumed that this difference is not enough to compensate for the more frequent cystectomies or greater risk of dying from cancer. Further, in the test arm at stage 3, 12 and 24 months MA urine analysis took place in addition to cystoscopy. Cystoscopy has by definition a high sensitivity. Therefore, at these stages the MA urine analyses might be superfluous and its costs can be saved here, i.e. in practice urologists should use MA as a substitute rather than as an adjunct for bladder cancer detection and follow-up. Another point is that MA blood analysis was required to provide reference values for MA urine analyses. The cost of MA blood analysis was incurred only once, at the start of surveillance. Over the course of, e.g. 10 years instead of 2 years of surveillance, there will hence be less difference in total costs between the arms.

Our study had some further limitations, primarily related to various assumptions that had to be made in the model to make the model manageable. For example, we assumed that progression only takes place after recurrence. However, it is conceivable that progression occurs directly from the health state 'Well'. Based on data from the present study, we can assume that MA is more sensitive for progression than for recurrence. In all, this would make the cost-effectiveness for MA worse. Finally, it is not immediately clear how to exactly translate our cost estimates in other countries or clinical practice routines. The costs might vary from one area to another in Europe, and in the USA, and the panel of tested markers can be improved. Further prospective studies in a larger international population are therefore necessary.

Some recommendations can be made. The costs of MA (blood and urine analysis) were higher than the costs of cystoscopy. The large difference in cost is caused by material as well as personnel costs. Further automation of MA testing might lead to mass production and cost reduction. Research is needed to find out how much the cost per head will decrease due to automation. According to the model, the current MA markers were not sensitive enough compared with cystoscopy. Better MA markers should be found to obtain better cost-effectiveness. Sensitive markers of particular interest are those that do not require comparison with a blood sample of the patient for reference values. Notably, based on data from the present study, it is conceivable that awareness of a positive MA outcome might have prompted a higher detection rate of UC recurrences by cystoscopy. Indeed, separate analysis of the patients of the MA arm showed an improved cross-sectional sensitivity (70\%) of the MA test [8]. Recent developments suggest other DNA-based tests as alternatives, such as detection of FGFR3 mutations (present in most NMI-UC) and assays for detecting tumour-associated methylation of genes of interest [14-18], as these tests are cheaper than MA in material and personnel costs.

In conclusion, over the 2 years of surveillance in which cystoscopy was partly replaced by currently available urinary MA to reduce patient burden, it can only provide a costeffective alternative to conventional surveillance if the MA urine test had slightly better sensitivity and its costs were reduced.

\section{ACKNOWLEDGEMENTS}

We appreciate the support of all urologists and pathologists who participated in this study, which was sponsored by the Efficiency Research program of the Netherlands Organization for Health Research and Development (grant number 945-02-046).

\section{CONFLICT OF INTEREST}

None declared.

\section{REFERENCES}

1 Kirkali Z, Chan T, Manoharan M et al. Bladder cancer: epidemiology, staging and grading, and diagnosis. Urology 2005; 66 (6 Suppl. 1): 4-34

2 Almallah $\mathrm{YZ}$, Rennie CD, Stone J, Lancashire MJ. Urinary tract infection and patient satisfaction after flexible cystoscopy and urodynamic evaluation. Urology 2000; 56: 37-9

3 Denholm SW, Conn IG, Newsam JE, Chisholm GD. Morbidity following cystoscopy: comparison of flexible and rigid techniques. Br J Urol 1990; 66: 1524

4 Clark KR, Higgs MJ. Urinary infection following out-patient flexible cystoscopy. Br J Urol 1990; 66: 503-5

5 Ross JS, Cohen MB. Ancillary methods for the detection of recurrent urothelial neoplasia. Cancer 2000; 90: 75-86

6 Botteman MF, Pashos CL, Redaelli A, Laskin B, Hauser R. The health economics of bladder cancer: a comprehensive review of the published literature. Pharmacoeconomics 2003; 21 : 1315-30

7 Bartoletti R, Dal Canto M, Cai T et al. Early diagnosis and monitoring of superficial transitional cell carcinoma by 
microsatellite analysis on urine sediment. Oncol Rep 2005; 13: 531-7

8 van der Aa MN, Zwarthoff EC, Steyerberg EW et al. Microsatellite analysis of voided-urine samples for surveillence of low-grade non-muscleinvasive urothelial carcinoma: feasibility and clinical utility in a prospective multicenter study (Cost-Effectiveness of Follow-Up of Urinary Bladder Cancer Trial [CEFUB]). Eur Urol 2008 (in press)

9 Amira N, Mourah S, Rozet F et al. Noninvasive molecular detection of bladder cancer recurrence. Int J Cancer 2002; 101 : 293-7

10 Raman JD, Sosa RE, Vaughan ED Jr, Scherr DS. Pathologic features of bladder tumors after nephroureterectomy or segmental ureterectomy for upper urinary tract transitional cell carcinoma. Urology 2007; 69: 251-4

11 Gold MR, Siegel JE, Russel LB, Weinstein MC. Cost-effectiveness in Health and Medicine. New York: Oxford University Press, 1996

12 Grossman HB, Soloway M, Messing E et al. Surveillance for recurrent bladder cancer using a point-of-care proteomic assay. JAMA 2006; 295: 299-305

13 Lotan Y, Roehrborn CG. Costeffectiveness of a modified care protocol substituting bladder tumor markers for cystoscopy for the followup of patients with transitional cell carcinoma of the bladder: a decision analytical approach. J Urol 2002; 167: 75-9

14 Dulaimi E, Uzzo RG, Greenberg RE, AlSaleem T, Cairns P. Detection of bladder cancer in urine by a tumor suppressor gene hypermethylation panel. Clin Cancer Res 2004; 10: 1887-93

15 Rieger-Christ KM, Mourtzinos A, Lee PJ et al. Identification of fibroblast growth factor receptor 3 mutations in urine sediment DNA samples complements cytology in bladder tumor detection. Cancer 2003; 98: 737-44

16 Miyake M, Sugano K, Kawashima K et al. Sensitive detection of FGFR3 mutations in bladder cancer and urine sediments by peptide nucleic acidmediated real-time PCR clamping. Biochem Biophys Res Commun 2007; 362: 865-71

17 van Oers JM, Lurkin I, van Exsel AJ et al. A simple and fast method for the simultaneous detection of nine fibroblast growth factor receptor 3 mutations in bladder cancer and voided urine. Clin Cancer Res 2005; 11: 7743-8

18 van Rhijn BW, Lurkin I, Chopin DK et al. Combined microsatellite and FGFR3 mutation analysis enables a highly sensitive detection of urothelial cell carcinoma in voided urine. Clin Cancer Res 2003; 9: 257-63

Correspondence: Esther W. de Bekker-Grob, Department of Public Health, Erasmus MC, PO Box 2040, 3000 CA Rotterdam, the Netherlands.

e-mail: e.debekker@erasmusmc.nl

Abbreviations: UC, urothelial carcinoma; (N)MI, (non-) muscle-invasive; TUR, transurethral resection; UUT, upper urinary tract; MA, microsatellite analysis; CEFUB, Cost-effectiveness of Follow-up of urinary Bladder Cancer.

\section{APPENDIX 1}

Decision-analytic model for bladder cancer surveillance.

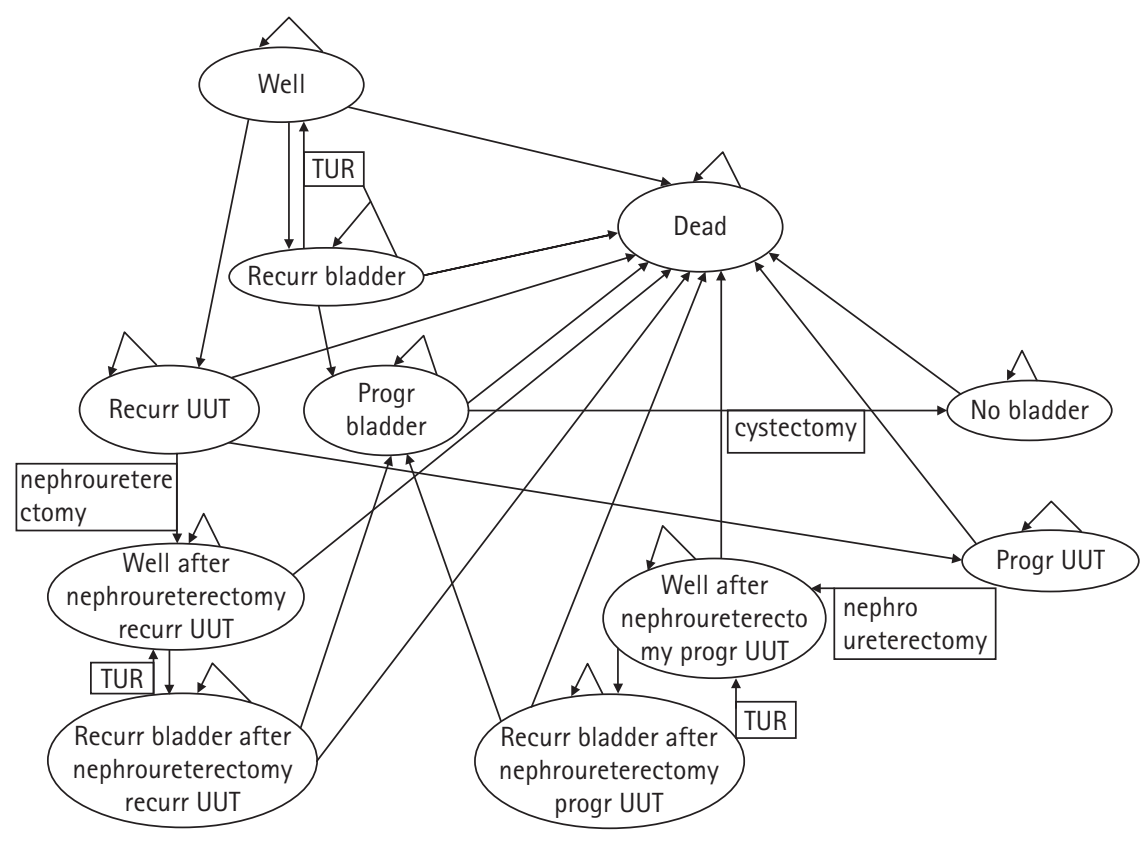

